\title{
Neuropeptide expression and morphometric differences in crushed alveolar inferior nerve of rats: Effects of photobiomodulation
}

\author{
Daniel Oliveira Martins ${ }^{1}$ Fabio Martinez dos Santos ${ }^{1,2}$ - Adriano Polican Ciena ${ }^{1,3}$. \\ Ii-sei Watanabe ${ }^{1}$ - Luiz Roberto G. de Britto ${ }^{4}$. José Benedito Dias Lemos ${ }^{5}$. \\ Marucia Chacur ${ }^{1}$
}

Received: 14 December 2016/Accepted: 23 February 2017 / Published online: 17 March 2017

(C) Springer-Verlag London 2017

\begin{abstract}
Inferior alveolar nerve (IAN) injuries may occur during various dental routine procedures, especially in the removal of impacted lower third molars, and nerve recovery in these cases is a great challenge in dentistry. Here, the IAN crush injury model was used to assess the efficacy of
\end{abstract}

Daniel Oliveira Martins

martinsd@usp.br

Fabio Martinez dos Santos

fabiomartinez@uninove.br

Adriano Polican Ciena

adrianociena@usp.br

Ii-sei Watanabe

watanabe@icb.usp.br

Luiz Roberto G. de Britto

britto@icb.usp.br

José Benedito Dias Lemos

jbdlemos@usp.br

Marucia Chacur

chacurm@icb.usp.br

1 Department of Anatomy, Institute of Biomedical Sciences, University of São Paulo, Av. Prof. Lineu Prestes, 2415, São Paulo, SP 05508-000, Brazil

2 University Nove de Julho, São Paulo, SP, Brazil

3 Institute of Biosciences, University Estadual Paulista Júlio de Mesquita Filho, Rio Claro, SP 13506-900, Brazil

4 Department of Physiology and Biophysics, Institute of Biomedical Sciences, University of São Paulo, Av. Prof. Lineu Prestes, 2524, São Paulo, SP 05508-000, Brazil

5 Department of Surgery, School of Dentistry, University of São Paulo, Av. Prof. Lineu Prestes, 2227, São Paulo, SP 05508-000, Brazil photobiomodulation (PBM) in the recovery of the IAN in rats following crushing injury (a partial lesion). Rats were divided into four experimental groups: without any procedure, IAN crush injury, and IAN crush injury with PBM and sham group with PBM. Treatment was started 2 days after surgery, above the site of injury, and was performed every other day, totaling 10 sessions. Rats were irradiated with GaAs Laser (Gallium Arsenide, Laserpulse, Ibramed Brazil) emitting a wavelength of $904 \mathrm{~nm}$, an output power of $70 \mathrm{mWpk}$, beam spot size at target $\sim 0.1 \mathrm{~cm}^{2}$, a frequency of $9500 \mathrm{~Hz}$, a pulse time $60 \mathrm{~ns}$, and an energy density of $6 \mathrm{~J} / \mathrm{cm}^{2}$. Nerve recovery was investigated by measuring the morphometric data of the IAN using TEM and by the expression of laminin, neurofilaments (NFs), and myelin protein zero (MPZ) using Western blot analysis. We found that IAN-injured rats which received PBM had a significant improvement of IAN morphometry when compared to IAN-injured rats without PBM. In parallel, all MPZ, laminin, and NFs exhibited a decrease after PBM. The results of this study indicate that the correlation between the peripheral nerve ultrastructure and the associated protein expression shows the beneficial effects of PBM.

Keywords Molecular changes · Alveolar inferior nerve · Photobiomodulation · Myelin sheath $\cdot$ Rat

\section{Introduction}

The improvement of peripheral nerve regeneration does not just rely only on the advancement of new microsurgical techniques and/or transplantation strategies and devices but also on the associated use of therapeutic protocols which can enhance the effectiveness of nerve regeneration [1]. In this way, non-invasive approaches have also been elaborated to facilitate the process. 
Different tools, such as electricity, electromagnetism, therapeutic ultrasound, and photobiomodulation (PBM), have all been employed for physical therapy purposes to improve the peripheral process of nerve repair $[2,3]$.

The favorable outcome of peripheral nervous system regeneration has been linked with changes of the microenvironment, especially of the extracellular matrix (ECM) components. ECM of the regenerating nerve is not simply a passive adhesive scaffold for regrowth, but actually, ECM molecules can synergistically signal with growth factors and growth cone molecules to influence regrowth. The ECM receptors, integrins, are essential not only for adhesive interactions but also for transducing growth related-signals [4].

Understanding how cells of the peripheral nervous system have an effective role in myelin formation in peripheral nerve regeneration after injury is a major challenge to create new therapies for nerve repair. The expression of the myelin protein zero (MPZ) is specific for the myelin sheath of the peripheral nervous system and is used as an indicator for the process of myelination [5]. Laminin is an important factor for neuritic outgrowth and for the facilitation of the formation of growth cones. After peripheral nerve injury, laminin has its expression significantly increased in damaged areas [6]. Laminin is mainly produced by Schwann cells, and it is widely expressed in the peripheral nervous system. As an ECM adhesion protein, laminin appears to be an important molecule for the orientation of axons in vivo [7]. Another component of the ECM that influences regrowth is neurofilaments (NFs) that are the major intermediate filaments of neurons and provide structural support to the neuron. They are formed by three subunits, NFL, NFM, and NFH (light, medium, and heavy) [8]. NFs are neuronal-specific intermediate filaments with important roles in the development and maintenance of axonal structure and constitute a resident axonal matrix. Other function of NFs is the control of axonal caliber, which is important in relation to its conduction speed of impulses down the axon [9]. The highly regulated expression of NF proteins during axon outgrowth suggests that NFs are important for axon development. In addition, loss of NFs can change specific parameters of neuritic growth dynamics [10].

Neurotrophic factors are vital to promote neural regeneration. Neurotrophins improve neuronal survival and regeneration of nerve fibers after spinal cord injury [11-13]. Among them, nerve growth factor (NGF) plays a critical role promoting proliferation and differentiation of neurons and modulates the repair of injured nerves [14]. In addition, NGF can promote axonal extension and myelination in proximal stumps of transected peripheral nerves in rats [15]. We have previously demonstrated that PBM increased the protein level of NGF after inferior alveolar nerve (IAN) injury, which was correlated to an improvement of the pain-related behavior [16].

The aim of this study was to verify whether PBM applied to injured IAN in rats is able to modify the microenvironment of the injury site by the analysis of the expression of some components that influence nerve regeneration, such as MPZ, laminin, and NFs. We also verified the ultrastructural morphology of IAN after injury and after PBM.

\section{Materials and methods}

\section{Animals}

A total of 40 specific pathogen-free male Wistar rats, weighing between 200 and $220 \mathrm{~g}$ ( 2 months old), were used in all experiments $(n=40 ; 5$ per group to Western blot and 5 per group to TEM). The animals were maintained on a $12: 12 \mathrm{~h}$ light/dark cycle. All procedures were approved by the Institutional Animal Care Committee of the University of São Paulo (protocol number 150-book number 02/2010). Efforts were made to minimize the number of animals used and their suffering [17].

\section{Surgical procedure}

\section{Crushing of the inferior alveolar nerve}

IAN crush was performed as described previously [16]. Briefly, rats were anesthetized with an intraperitoneal injection of a mixture of ketamine $(5 \mathrm{mg} / 100 \mathrm{~g}$ body weight, ip) and xylazine ( $1 \mathrm{mg} / 100 \mathrm{~g}$ body $\mathrm{w}$, ip). The skin was sterilized around the inferior jaw with povidone-iodine, and a small incision was made on the surface of the facial skin over the masseter muscle about $1 \mathrm{~cm}$ long. The alveolar bone was carefully removed, and the IAN was exposed on the right side. The IAN was crushed for $30 \mathrm{~s}$, and the incision was sutured. A false-operated animals group (sham), without nerve injury, was also organized. The rats were monitored until they fully recovered and then brought back to the animal facility.

\section{Photobiomodulation}

The treatment was similar to that used in previous study [16]. Briefly, rats were randomly divided into four groups (five rats per group). Two of these groups were irradiated with GaAs laser (Gallium Arsenide, Laserpulse-Laser, Ibramed Brazil) emitting a wavelength of $904 \mathrm{~nm}$, an output power of $70 \mathrm{mWpk}$, beam spot size at target $\sim 0.1 \mathrm{~cm}^{2}$, a frequency of $9500 \mathrm{~Hz}$, a pulse time $60 \mathrm{~ns}$, and an energy density of $6 \mathrm{~J} / \mathrm{cm}^{2}$. The treatment started after 2 days of IAN injury or in sham animals. The laser treatment was performed every other day, involving 10 sessions. After sterilization, laser was lightly placed on the skin surface directly above the neural pathway of IAN, without skin contact. A point near the temporomandibular joint and four points in the region of the inferior jaw 
body were irradiated, for $18 \mathrm{~s}$ per point, in intervals of $30 \mathrm{~s}$, and each session had duration of $4 \mathrm{~min}$.

\section{Tissue preparation}

\section{Immunoblotting}

Inferior alveolar nerves (four for each condition) were rapidly collected and frozen in liquid nitrogen and stored at $-80{ }^{\circ} \mathrm{C}$ until use, and $100 \mu \mathrm{L}$ extraction buffer (100 mM Tris, $\mathrm{pH} 7.4$, $10 \mathrm{mM}$ EDTA, $2 \mathrm{mM}$ PMSF, and $10 \mu \mathrm{g} / \mathrm{mL}$ aprotinin) was added in the tube. The TG was then homogenized using an ultrasonic processor (Sonics \& Materials). The extracts were then centrifuged at $12,000 \mathrm{rpm}$ at $4{ }^{\circ} \mathrm{C}$ for $20 \mathrm{~min}$ to separate the insoluble material. Protein concentration in the supernatants was determined using Bradford method with albumin as a standard (Bio-Rad) [18]. The whole extracts were treated with Laemmli sample buffer containing dithiothreitol and boiled for 5 min before loading onto $12 \%$ acrylamide SDS gels (Bio-Rad, USA) and electrophoretically transferred to nitrocellulose membranes (Millipore) at $120 \mathrm{~V}$ for $1.5 \mathrm{~h}$ using a Trans-Blot Cell System (Bio-Rad). Non-specific protein binding to the nitrocellulose membrane was reduced by preincubation for $2 \mathrm{~h}$ at $22^{\circ} \mathrm{C}$ in blocking buffer containing (5\% non-fat dry milk). The nitrocellulose membranes were incubated overnight at $4{ }^{\circ} \mathrm{C}$ with an anti-NF (monoclonal antibody cocktail; MAB2300-Millipore Corporation), a polyclonal anti-MPZ (ab31851; Abcam), and a monoclonal anti-laminin (Sigma); diluted 1:1000; and then washed for $30 \mathrm{~min}$. The membranes were subsequently incubated with a peroxidase-conjugated secondary antibody anti-rat (Zymed) or anti-rabbit (GE Healthcare), diluted 1:5000 in TTBS with $1 \%$ non-fat milk for $2 \mathrm{~h}$, and processed for enhanced chemiluminescence to visualize the immunoreactive bands in the Storm 860 Molecular Imager. An anti- $\beta$-actin antibody (Sigma) was used to quantify $\beta$-actin as a loading control. Densitometric analysis of the bands was performed using NIH-Scion Image 4.0.2, and protein expression was quantified using the ImageJ software (Scion Corporation, Release Beta 3b, NIH).

\section{Transmission electron microscopy}

The TEM protocol was performed as described previously $[19,20]$. So, briefly, a $3-\mathrm{mm}^{3}$ segment of the IAN distal to the site of crush injury was used for the ultrastructural analysis. Ultrathin $60 \mathrm{~nm}$ sections were prepared according to Watanabe and Yamada method [21]. The sections were examined, and images were collected under a transmission electron microscope (Jeol 1010, Tokyo). At last, standardized relative quantification of morphometric analysis was performed using Image Java software (Bethesda, MD, USA) [22] in order to measure the nerve morphometric parameters of interest. We analyzed the diameters of nerve fibers, axon diameters, and myelin sheath thickness, for naive, IAN injury and IAN injury + PBM groups. The G-ratios were also calculated by dividing the diameters of the axon by the fiber diameters [20].

\section{Statistical analysis}

The data are presented as means \pm SEM or means \pm SD (for morphometric analysis) and were analysed by the ANOVA followed by the pairwise post-hoc Tukey's HSD test, and $p \leq 0.05$ was considered statistically significant [23].

\section{Results}

\section{Effects of PBM upon protein expression in the IAN}

Single positive bands were observed in IAN extracts for laminin and MPZ. On the other hand, we observed three bands corresponding to the three subunits of NFs in nerve extracts: NFL, NFM, and NFH. Our results demonstrate an increase of laminin expression in IAN-lesioned animals (IAN injury) comparing with naive rats, used as a control and determined as $100 \%$. Densitometric analysis revealed that laminin increased by about $45 \%(p \leq 0.05)$ above the control. In contrast, rats under PBM (IAN injury + PBM) exhibited a decrease of expression in relation to non-treated rats (ca. $57 \%$ above the control) ( $p \leq 0.001)$. The control groups (naive and sham) did not show statistical differences in laminin level (Fig. 1).

The data for MPZ also revealed an increase of expression protein level (ca. 20\% above the control) $(p \leq 0.05)$ following IAN crush when compared to naive group. In turn, there was a decrease (ca. 74\% below the control) $(p \leq 0.0001)$ in rats with
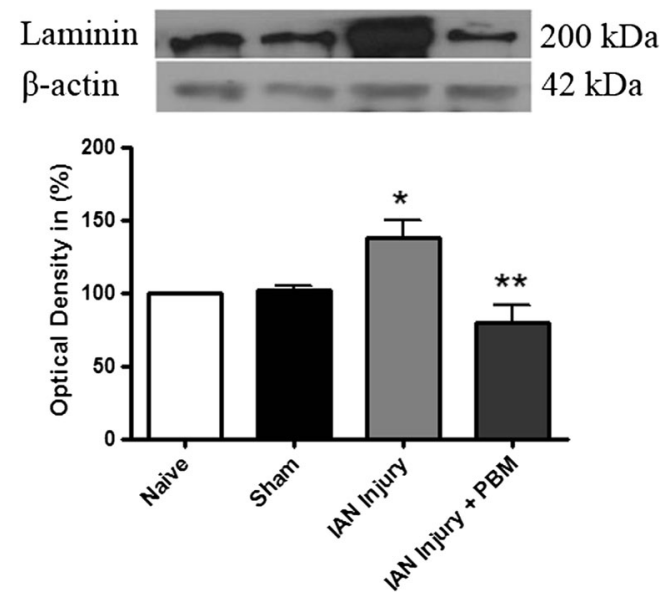

Fig. 1 Densitometric analysis of laminin protein levels. Data are normalized in relation to data from naive rats, which were taken as $100 \%$. Data are reported as mean $\pm \mathrm{SEM}$ of five animals per group. $* p \leq 0.05$ compared to control naive group and $* * p \leq 0.001$ compared to IAN injury group 

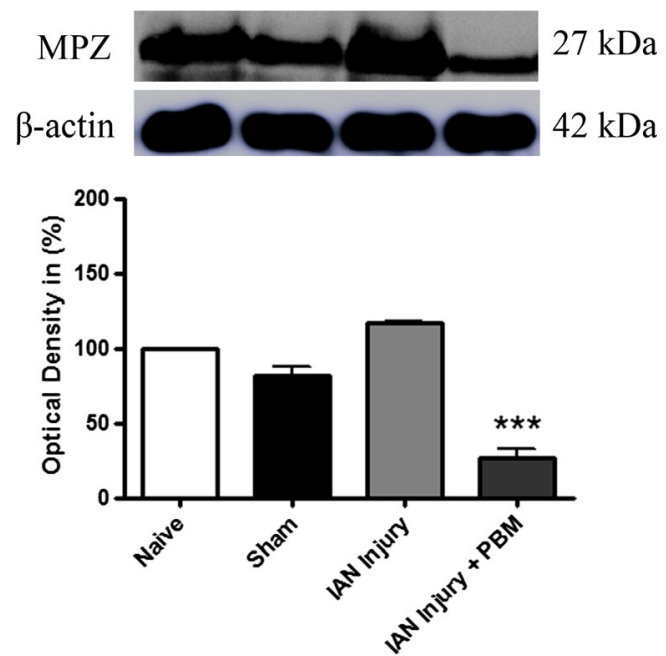

Fig. 2 Densitometric analysis of MPZ protein levels. Data are normalized in relation to naive rats; data are taken as $100 \%$ in relation to data from naive rats. Data are reported as mean \pm SEM of five animals per group. $* p \leq 0.05$ compared to control animals and $* * * p \leq 0.001$ compared to IAN injury group

treatment (IAN injury + PBM). No differences were observed between naive and sham animals, used as controls (Fig. 2).

In the immunoblotting results for NFs, we observed three bands (200-160-68 kDa, corresponding to NFH, NFM, and NFL respectively) obtained from extracts of IAN. The densitometric analysis for all NFs evaluated did not show changes in expression levels in the IAN-injured group in comparison with the control group. Otherwise, afterwards, 10 sessions of PBM the IAN injury + PBM group showed a decrease in the protein expression for all NFs evaluated when compared to the bands of the naive group NFH, ca. $60 \%$ below the control, $p \leq 0$. 001; NFM, ca. $52 \%$ below the control, $p \leq 0$. 05; and NFL, ca. $62 \%$ below the control, $p \leq 0$. 001. Statically, naive and sham groups (used as controls) showed no differences (Fig. 3).

\section{Transmission electron microscopy data}

Electron microscopic analysis ( $3 \mathrm{~mm}$ distal to the crushing) showed that the IAN from the control group (naive) exhibited a normal morphology of axons and Schwann cells (Fig. 4a, b). In the IAN injury group (20 days after the injury), the myelin sheath has been seriously damaged. Vacuolization occurred in the axonal cytoplasm (Fig. 4c, d). However, the damaged myelin sheath appears to recover, and axons and Schwann cells showed normal morphology after treatment with PBM for 10 sessions in the IAN injury + PBM group (Fig. 4e, f). The morphometric data obtained for the distal segments of the IAN of the three groups are presented in Fig. 5. In the myelin sheath thickness measure, we observed that the IAN of the naive animals has presented a thicker myelin sheath (1.59 $\pm 0.18 \mu \mathrm{m})$ comparing with those the injured animals group
Fig. 3 Densitometric analysis of NF protein levels. Data are normalized in relation to those from naive rats. Data are reported as mean $\pm \mathrm{SEM}$ of five animals per group. ${ }^{*} p \leq 0.05$ compared to control animals and $* * p \leq 0.001$ compared to IAN injury group
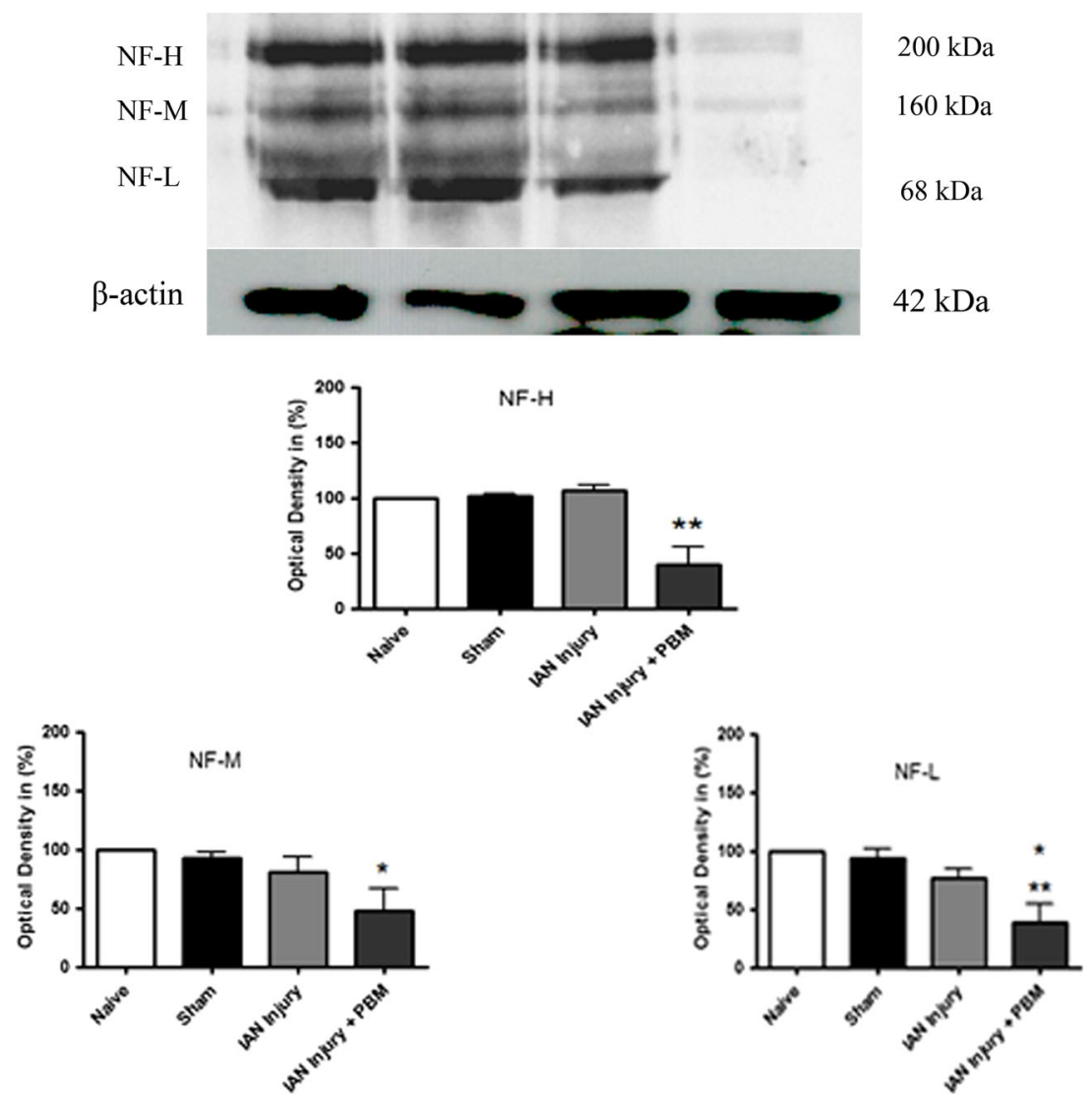


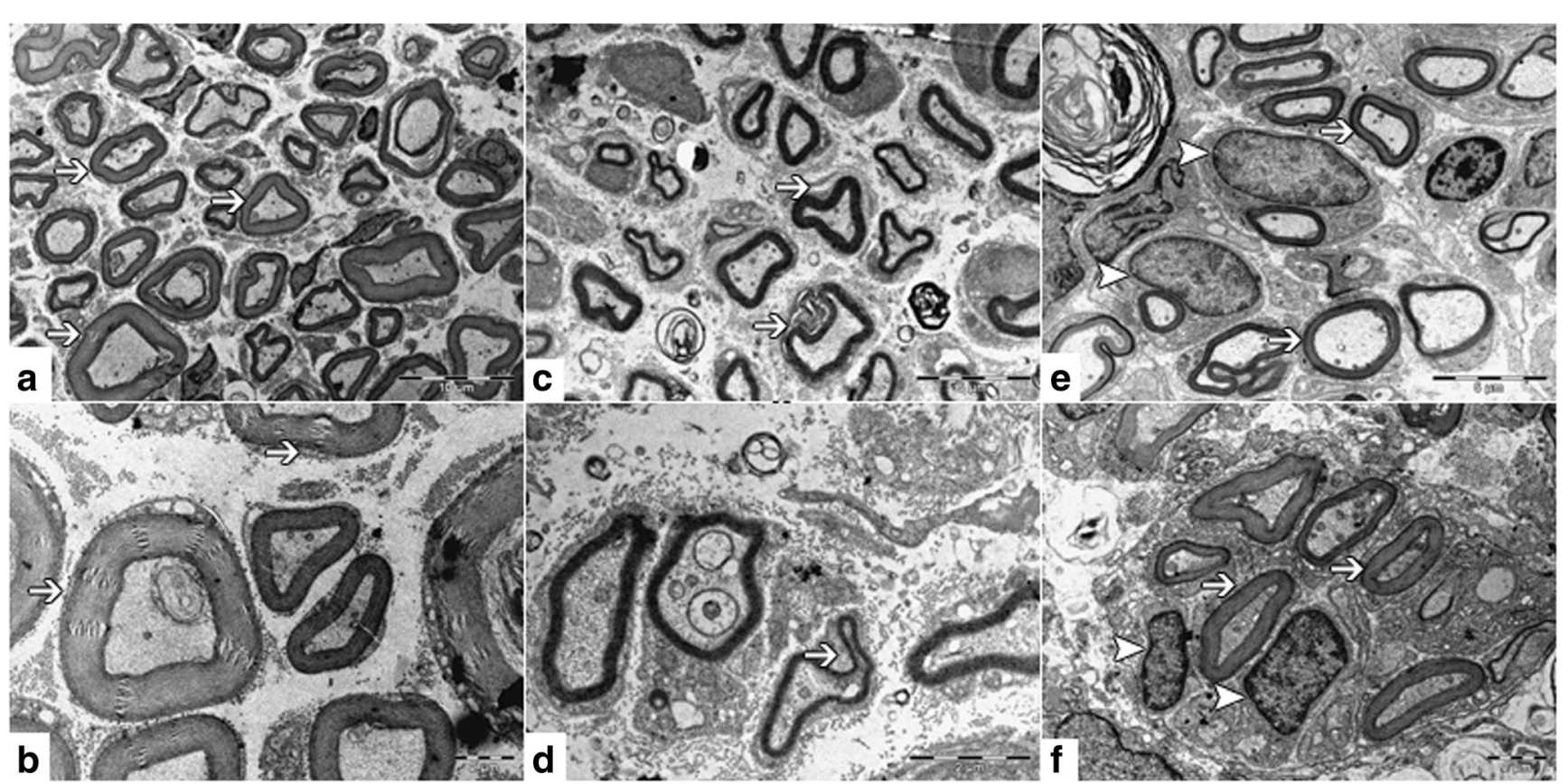

Fig. 4 Transmission electron photomicrographs of the IAN. The ultrastructural morphology of IAN was observed in different groups. Nerves were examined by transmission electron microscopy. Representative images are shown. a, b IAN intact fiber, with a regular distribution of the unmyelinated and myelinated fibers. c, d IAN after 20 days of injury; the structure of the nerve was severely damaged as characterized by Wallerian degeneration, showing axonal degeneration. e, f IAN after 20 days of injury and treatment with low level laser; regenerated unmyelinated and myelinated fibers and in the process regeneration are visible. Arrows highlight the myelin sheath in $\mathbf{a}, \mathbf{b}$ (control group) demonstrating a thicker and well-organized myelin sheath compared with the IAN injury group $(\mathbf{c}, \mathbf{d})$. e, f Effects of PBM for regenerating nerve fibers. The thickness of the myelin sheet showed significant difference to the IAN injury group. Arrowheads highlight the Schwann cell nuclei, demonstrating the regeneration process. Scale bars: $\mathbf{a}, \mathbf{c}, \mathbf{e} 10 \mu \mathrm{m} ; \mathbf{b}, \mathbf{d}, \mathbf{f} 2 \mu \mathrm{m}$
$(0.41 \pm 0.06 \mu \mathrm{m})$. In the IAN-injured animals, the rats in which PBM was performed, we saw a significantly improvement in the thick of myelin sheaths $(0.60 \pm 0.08 \mu \mathrm{m})$ when compared with the untreated injured group $(0.41 \pm 0.06 \mu \mathrm{m})$. The other morphometric parameters evaluated in this study, nerve fiber diameter and axon diameter, show similar results, and IAN-injured untreated group presented all parameters worse than the other groups (Fig. 5).

\section{Discussion}

Experimental animal models have been created to improve the understanding of the pathophysiological mechanisms of post-traumatic nerve repair and its complications. Photobiomodulation became the mostly common tool used for non-invasive therapy purposes. We recently showed that PBM improve the functional recovery of IAN crushed accessed by the Von Frey test, evaluating the head escape threshold to mechanical stimulation of the mental skin, according to a modification of the method described by Chaplan and Yonehara [24, 25]. After the crush injury to the rat's IAN, the escape threshold to mechanical stimulation of the mental skin was significantly lower than before surgery and remains until the last time evaluated. When the animals were submitted to the PBM, the threshold value was significantly increased compared to injured group, returning to normal levels (for more information see ref. [16]). So, the purpose of this study was to evaluate if PBM application on the site of injury is able to modify the cellular and molecular microenvironment with a focus especially on protein myelin zero, laminin, and neurofilaments.

Following peripheral nerve injury, Schwann cells (SCs) in association with the degenerating axons dedifferentiate and proliferate, remove myelin and axonal debris, and align to support axonal regeneration [26]. The proregenerative capabilities of SCs have been exploited to enhance regeneration in the central nervous system [27, 28], an effect suggested to depend on ECM proteins and neurotrophic factors expressed by SCs $[26,29]$.

The ECM has been implicated in myelination in the peripheral nervous system, and laminins are components of the ECM that appear to be especially important. Studies in cell culture have suggested that laminin plays a critical role in myelination $[30,31]$. Laminin synthesis by SCs is essential for myelination. During development and regeneration, specific molecules regulate each stage of SC development [32] and laminin is one of these molecules [33]. Without laminin, Schwann cells can migrate along 
a

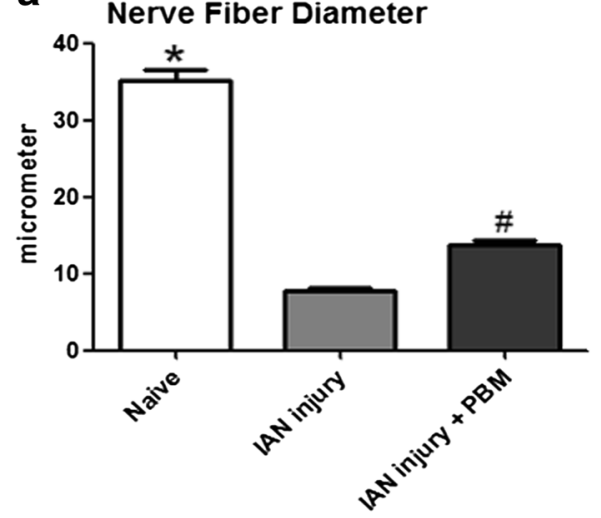

C

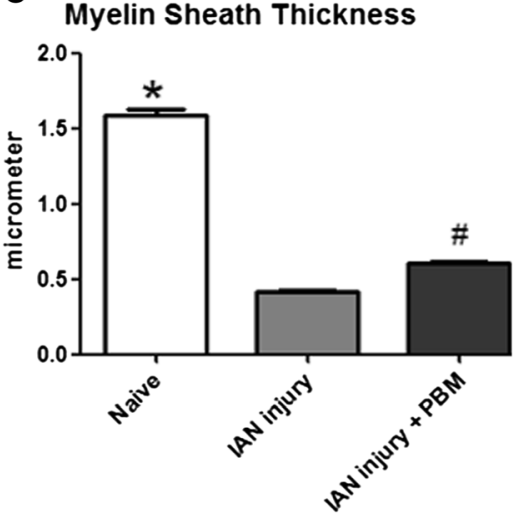

Fig. 5 Morphometric analysis of IAN. The nerve fiber diameter, axon diameter, myelin sheath thickness, and the G-ratios (calculated by dividing the diameter of the axon by the fiber diameter) in all groups were evaluated. Results are presented as means \pm SD. Asterisk indicates

the axons, populate the peripheral nerve, and proliferate normally, but cannot differentiate into a myelinating phenotype [7]. On the other hand, laminin can also regulate neurite outgrowth acting coordinately with GTPases [34], which are needed to control the contractile capability dependent on actin-myosin interactions [35]. In our study, we observed that IAN injury increased the expression of laminin, consistent with a study that showed that laminin is highly increased in the site of injury after damage of peripheral nerves [6]. After PBM, the level of laminin expression decreased. Laminin is upregulated in developing nerves but is normally low in mature nerves [36], and it plays a critical role in axonal sorting, an essential step in the development of the peripheral nervous system [37, 38]. Another important protein in the remyelinating process is MPZ, which is the most abundant protein of the peripheral myelin sheath [39]. The expression of MPZ is specific for the myelin sheath in the peripheral nervous system and is used as an indicator for the myelination process [5]. The same pattern observed here for laminin was observed for MPZ, with an increase in injured animals. MPZ may play a critical role in response to nerve injury; once after PBM, there was a decrease in protein expression for both laminin and MPZ.

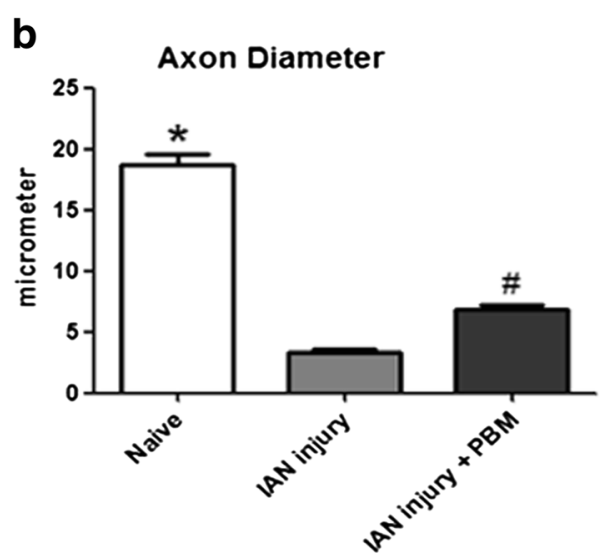

\begin{tabular}{lllll}
\hline & Nerve Fiber & Axon & \multicolumn{2}{l}{ Myelin sheath } \\
Group & Diameter & Diameter & thickness & G-ratios \\
\hline Naive & $35.21 \pm 6.34$ & $18.67 \pm 3.91$ & $1.59 \pm 0.18$ & $0.53^{\circ}$ \\
IAN Injury & $7.85 \pm 2.73$ & $3.37 \pm 1.48$ & $0.41 \pm 0.06$ & 0.42 \\
IAN Injury+P8M & $13.67 \pm 4.18$ & $6.84 \pm 1.52$ & $0.60 \pm 0.08$ & $0.50^{*}$ \\
\hline
\end{tabular}

a significant difference between the naive and IAN injury groups; number sign indicates a significant difference between the IAN injury + PBM and IAN injury group. ${ }^{*} p \leq 0.001$ and $\# p \leq 0.05$

NFs are also markers of regeneration, and NF expression constitutes the initial evidence of nerve regeneration potential [40]. PBM changed the protein expression of all NFs evaluated here. The main known function for NFs is to increase axonal caliber of myelinated axons and, consequently, its conduction velocity. They also contribute to the dynamic properties of the axonal cytoskeleton during neuronal differentiation, axonal growth, guidance, and regeneration [41]. The examination by transmission electron microscopy of nerves that did not receive PBM demonstrated that the crushed nerve exhibited various degrees of degeneration in the 20-day period subsequent to the crush injury. However, in the rats in which PBM was performed, there was Schwann cell proliferation and immature myelin formation.

Several molecules are known to play an important role in axonal regeneration, but the way they interact with each other is still unclear. A systematic review highlighted the effectiveness of PBM for the treatment of peripheral nerve lesions. Suggesting that PBM may promote higher metabolism in neurons and a better ability to produce myelin due the release of certain growth factors and cytokines by various types of cells which can be modulated by laser irradiation [42]. This study 
shows that photobiomodulation was able to alter the microenvironment of the injury site via modulation of key elements that are able to create a permissive environment for axons to grow and influence regeneration.

The present findings show changes of molecules that play an important role during peripheral nerve regeneration and confirmed that the selected PBM pattern was sufficient to accelerate the rate of recovery of injured peripheral nerve. Finally, based on the expression of nerve regeneration markers, morphological parameter changes, and previous behavioral tests, we showed that the PBM could offer a potential proregenerative environment and beneficial results to peripheral nerve repair. We hope that these results can be used to improve the current treatments using PBM in new integrated treatment strategies.

Acknowledgements The authors are grateful to Sônia Regina Yokomizo for her valuable help.

Authors' contributions All authors made substantial contributions to the following tasks of research: initial conception (Martins D.O., Britto L.R.G., Lemos J.B.D., Chacur M.), design (Martins D.O., Britto L.R.G., Lemos J.B.D., Chacur M), provision of resources (Chacur M), collection of data (Martins D.O, Santos F.M.,, Ciena A.P., Watanabe I.), analysis and interpretation of data (Martins D.O., Chacur M., Ciena A.P., Watanabe I.), writing the first draft of the paper or important intellectual content (Martins D.O, Santos F.M.), and revision of paper (Martins D.O., Chacur M., Britto L.R.G.).

\section{Compliance with ethical standards}

Ethics approval and consent to participate All procedures were approved by the Institutional Animal Care Committee of the University of São Paulo (protocol number 150/2010) and performed in accordance with the guidelines for the ethical use of conscious animals in pain study published by the international association for the study of pain.

Competing interests The authors declare that they have no competing interests.

Funding FAPESP 2010/20026-6; 2012/05840-4; 2014/24533-0

\section{References}

1. Raimondo S et al (2011) Perspectives in regeneration and tissue engineering of peripheral nerves. Ann Anat 193(4):334-40

2. Mendonca AC, Barbieri CH, Mazzer N (2003) Directly applied low intensity direct electric current enhances peripheral nerve regeneration in rats. J Neurosci Methods 129(2):183-90

3. Anders JJ, Geuna S, Rochkind S (2004) Phototherapy promotes regeneration and functional recovery of injured peripheral nerve. Neurol Res 26(2):233-9

4. Tucker BA, Mearow KM (2008) Peripheral sensory axon growth: from receptor binding to cellular signaling. Can J Neurol Sci 35(5): 551-66

5. Wang X et al (2011) Schwann-like mesenchymal stem cells within vein graft facilitate facial nerve regeneration and remyelination. Brain Res 1383:71-80
6. Thompson DM, Buettner HM (2001) Schwann cell response to micropatterned laminin surfaces. Tissue Eng 7(3):247-65

7. Chen ZL, Strickland S (2003) Laminin gamma1 is critical for Schwann cell differentiation, axon myelination, and regeneration in the peripheral nerve. J Cell Biol 163(4):889-99

8. Yuan A et al (2009) Neurofilaments form a highly stable stationary cytoskeleton after reaching a critical level in axons. J Neurosci 29(36):11316-29

9. Al-Chalabi A, Miller CC (2003) Neurofilaments and neurological disease. Bioessays 25(4):346-55

10. Walker KL et al (2001) Loss of neurofilaments alters axonal growth dynamics. J Neurosci 21(24):9655-66

11. Shao Y et al (2002) Effect of nerve growth factor on changes of myelin basic protein and functional repair of peripheral nerve following sciatic nerve injury in rats. Chin J Traumatol 5(4):237-40

12. Boyce VS et al (2007) Neurotrophic factors promote and enhance locomotor recovery in untrained spinalized cats. J Neurophysiol 98(4):1988-96

13. Sasaki M et al (2009) BDNF-hypersecreting human mesenchymal stem cells promote functional recovery, axonal sprouting, and protection of corticospinal neurons after spinal cord injury. J Neurosci 29(47):14932-41

14. Petruska JC, Mendell LM (2004) The many functions of nerve growth factor: multiple actions on nociceptors. Neurosci Lett 361(1-3):168-71

15. Zochodne DW (2000) The microenvironment of injured and regenerating peripheral nerves. Muscle Nerve Suppl 9:S33-8

16. de Oliveira Martins D et al (2013) Laser therapy and pain-related behavior after injury of the inferior alveolar nerve: possible involvement of neurotrophins. J Neurotrauma 30(6):480-6

17. Zimmermann M (1983) Ethical guidelines for investigations of experimental pain in conscious animals. Pain 16(2):109-10

18. Bradford MM (1976) A rapid and sensitive method for the quantitation of microgram quantities of protein utilizing the principle of protein-dye binding. Anal Biochem 72:248-54

19. Ciena AP et al (2012) Fine structure of myotendinous junction between the anterior belly of the digastric muscle and intermediate tendon in adults rats. Micron 43(2-3):258-62

20. da Silva JT et al (2015) Neural mobilization promotes nerve regeneration by nerve growth factor and myelin protein zero increased after sciatic nerve injury. Growth Factors 33(1):8-13

21. Watanabe I, Yamada E (1983) The fine structure of lamellated nerve endings found in the rat gingiva. Arch Histol Jpn 46(2):173-82

22. Schneider CA, Rasband WS, Eliceiri KW (2012) NIH Image to ImageJ: 25 years of image analysis. Nat Methods 9(7):671-5

23. Snedecor GW, Sokal RR, Rohlf FJ (1946) Statistical methods biometry. 4 ed. Ames, ed. W.H. Freeman \& Co. New York: Owa State University Press. p.859

24. Chaplan SR et al (1994) Quantitative assessment of tactile allodynia in the rat paw. J Neurosci Methods 53:55-63

25. Yonehara N, Kudo C, Kamisaki Y (2003) Involvement of NMDAnitric oxide pathways in the development of tactile hypersensitivity evoked by the loose-ligation of inferior alveolar nerves in rats. Brain Res 963(1-2):232-43

26. Chen ZL, Yu WM, Strickland S (2007) Peripheral regeneration. Annu Rev Neurosci 30:209-33

27. Fortun J, Hill CE, Bunge MB (2009) Combinatorial strategies with Schwann cell transplantation to improve repair of the injured spinal cord. Neurosci Lett 456(3):124-32

28. Toft A et al (2013) A comparative study of glial and non-neural cell properties for transplant-mediated repair of the injured spinal cord. Glia 61(4):513-28

29. Webber CA et al (2011) Schwann cells direct peripheral nerve regeneration through the Netrin-1 receptors, DCC and Unc5H2. Glia 59(10):1503-17 
30. Podratz JL, Rodriguez E, Windebank AJ (2001) Role of the extracellular matrix in myelination of peripheral nerve. Glia 35(1):35-40

31. Tsiper MV, Yurchenco PD (2002) Laminin assembles into separate basement membrane and fibrillar matrices in Schwann cells. J Cell Sci 115(Pt 5):1005-15

32. Mirsky R et al (2001) Regulation of genes involved in Schwann cell development and differentiation. Prog Brain Res 132:3-11

33. Uziyel Y, Hall S, Cohen J (2000) Influence of laminin-2 on Schwann cell-axon interactions. Glia 32(2):109-21

34. Rankin SL et al (2008) Neurotrophin-induced upregulation of p75NTR via a protein kinase C-delta-dependent mechanism. Brain Res 1217:10-24

35. Luo L (2002) Actin cytoskeleton regulation in neuronal morphogenesis and structural plasticity. Annu Rev Cell Dev Biol 18:601-35

36. Nakagawa $\mathrm{M}$ et al (2001) Schwann cell myelination occurred without basal lamina formation in laminin alpha2 chain-null mutant (dy3K/dy3K) mice. Glia 35(2):101-10
37. Yang D et al (2005) Coordinate control of axon defasciculation and myelination by laminin-2 and -8. J Cell Biol 168(4):655-66

38. Yu WM et al (2005) Schwann cell-specific ablation of laminin gammal causes apoptosis and prevents proliferation. J Neurosci 25(18):4463-72

39. Rotenstein L et al (2008) Characterization of the shark myelin Po protein. Brain Behav Evol 72(1):48-58

40. Varejao AS et al (2004) Functional and morphological assessment of a standardized rat sciatic nerve crush injury with a non-serrated clamp. J Neurotrauma 21(11):1652-70

41. Perrot R et al (2008) Review of the multiple aspects of neurofilament functions, and their possible contribution to neurodegeneration. Mol Neurobiol 38(1):27-65

42. Peplow PV, Chung TY, Baxter GD (2010) Laser photobiomodulation of proliferation of cells in culture: a review of human and animal studies. Photomed Laser Surg 28(Suppl 1):S3-40 